\title{
GOUÉRY, Franck \& JEANGÈNE VILMER, Jean-Baptiste. - Érythrée. Entre splendeur et isolement
}

Alain Gascon

\section{OpenEdition}

\section{Journals}

Édition électronique

URL : http://journals.openedition.org/etudesafricaines/20624

DOI : 10.4000 /etudesafricaines.20624

ISSN : 1777-5353

\section{Éditeur}

Éditions de l'EHESS

\section{Édition imprimée}

Date de publication : 1 avril 2017

Pagination : 184-188

ISBN : 978-2-7132-2688-5

ISSN : 0008-0055

\section{Référence électronique}

Alain Gascon, «GouérY, Franck \& Jeangène VILMER, Jean-Baptiste. - Érythrée. Entre splendeur et isolement », Cahiers d'études africaines [En ligne], 225 | 2017, mis en ligne le 01 avril 2017, consulté le 24 septembre 2020. URL : http://journals.openedition.org/etudesafricaines/20624 ; DOI : https:// doi.org/10.4000/etudesafricaines.20624

Ce document a été généré automatiquement le 24 septembre 2020.

(c) Cahiers d'Études africaines 


\title{
GOUÉRY, Franck \& JEANGÈNE VILMER, Jean-Baptiste. - Érythrée. Entre splendeur et isolement
}

\author{
Alain Gascon
}

\section{RÉFÉRENCE}

GOUÉRY, Franck \& JEANGÈNE VILMER, Jean-Baptiste. - Érythrée. Entre splendeur et isolement. Paris, Non Lieu, 2015, 168 p., bibl., ill.

1 Deux livres sur l'Érythrée, en français, paraissent au printemps 2015: c'est un événement qui mérite d'être célébré. En effet, les ouvrages qui traitent de l'ex-colonie italienne sont habituellement publiés en italien et, de plus en plus, en anglais. Mais ils sont rares, car les chercheurs ne sont pas vraiment les bienvenus dans ce pays. Ceci confirme, après bien d'autres signes, qu'Isayyas Afäwärqi, l'hérö̈que David érythréen dressé contre le Goliath éthiopien lié aux États-Unis puis à l'URSS, était un dictateur de la pire espèce. L'afflux constant de réfugiés aux frontières de l'Europe attire de plus en plus l'attention des médias sur la «dictature oubliée ». F. Gouéry et J.-B. Jeangène Vilmer ont réussi à pénétrer en Érythrée comme touristes et à tromper la vigilance d'une surveillance très étroite. Ainsi chaque matin, ils ne savaient pas s'ils obtiendraient l'autorisation de se rendre dans les sites « au programme » d'un circuit pourtant organisé par une société d'État.

2 Le premier livre, Érythrée. Entre splendeur et isolement, donne la priorité à des photographies de très belle facture. Le texte, bien qu'accompagné de notes et de références bibliographiques, est destiné à un « large » public. Les auteurs présentent les grandes périodes de l'histoire de la rive nord et montagneuse de la Corne de l'Afrique qui est devenue en 1890 la Colonià Eritrea. Conçue comme une porte d'entrée de l'Italie en Afrique, elle fut fermée par la victoire éthiopienne à Adwa en 1896. En 1936, elle servit, avec la Somalia italiana, de marchepied à la conquête de l'Impèro qui, en 1941, 
s'effondra sous les assauts de l'empire britannique, des Français et des Belges libres et de la résistance éthiopienne. La British Military Administration terminée en 1952, un vote de l'ONU fédéra l'Érythrée - dotée d'une constitution, d'un drapeau, d'une assemblée, d'un gouvernement, de deux langues officielles - à l'Éthiopie. La suite est connue : le gouvernement de Haylä Sellasé étouffa les institutions érythréennes et, en 1962, obtint par la menace et la prébende un vote de l'Assemblée érythréenne favorable à l'annexion à l'Éthiopie. Or, dès 1960, des Érythréens s'étaient soulevés dans les basses terres puis, au début des années 1970, dans les hautes terres. Les séparatistes contribuèrent à la chute du negus en 1974 et, alliés aux Tegréens et dirigés par Isayyas, délogèrent les troupes éthiopiennes d'Asmära en 1991. En 1993, l'Érythrée ratifia massivement le référendum d'indépendance et fut admise à l'ONU et à l'OUA. Dans un deuxième temps, les auteurs nous entrânent dans un parcours nostalgique à Asmära, à Massawa, à Kärän et dans les « vestiges de l'Empire italien ». Dans les derniers chapitres (après la page 121), le ton devient plus grave comme le montrent les titres : «Un État prédateur ", "Les droits humains » et "Fuir l'Érythrée ». F. Gouéry et J.-B. Jeangène Vilmer, en effet, tentent de concilier histoire, nostalgie et lucidité. Nous émettrons quelques réserves quant à un ouvrage dont les images, par leur qualité, tiennent la comparaison avec celles de Chris Kutschera ${ }^{14}$, un professionnel. Peut-on, toutefois, citer Monfreid, sans mentionner son soutien forcené à Mussolini? Les témoignages architecturaux à Asmära sont certes remarquables, mais doit-on omettre que les soussols de la station-service AGIP (p. 72) abritent un centre d'interrogatoire? On lit sur la « villa » (p. 104), de la « bucolique » (sic.) Kärän: Scuole Elementari. Le lecteur qui voudra approfondir la dernière partie aura intérêt à consulter leur deuxième ouvrage dont il va être question.

3 Le deuxième livre Érythrée. Un naufrage totalitaire, c'est du sérieux : le canon d'un des nombreux chars éthiopiens détruits par les Érythréens est pointé, en gros plan, sur le lecteur. Les auteurs y manifestent une double ambition : rédiger un essai théorique sur le totalitarisme et une analyse politique, en forme de portrait à charge d'Isayyas Afäwärqi et de sa « dérive » dictatoriale. Le cahier de photographies inclus, différent du livre précédent, montre de nombreux portraits, très émouvants, d'Érythréens ordinaires face aux aléas d'un quotidien difficile. Il manque une carte à plus grande échelle, centrée sur Asmära, localisant les centres où sont " éduqués " à la dure les jeunes Érythréens et Érythréennes. Dommage que les auteurs n'aient pas consulté un locuteur du tigrinya afin d'améliorer les transcriptions d'une langue, enseignée à l'INALCO et pourvue d'un syllabaire et non pas d'un alphabet (p.v). Dommage, également, quoi qu'ils en aient écrit, qu'ils aient délibérément ignoré les règles de l'onomastique érythréenne (p.v). La bibliographie très ambitieuse (pp. 295-316) comprend des ouvrages sur l'Érythrée et d'«autres sources » dont Aristote, pourquoi pas? Des renvois bibliographiques, souvent trois ou quatre à la fois au bas de chaque page, alourdissent la lecture.

Le livre adopte un plan en trois parties de taille inégale : «Les germes » de 1961 à 1991 (pp. 17-58), «La dérive» (pp.59-208) et enfin «Les failles» (pp. 209-290). Dans l'introduction (pp. 1-14), placée sous le patronage d'Orwell, F. Gouéry et J.-B. Jeangène Vilmer expliquent la signification du sous-titre : «un naufrage totalitaire ». Il s'agit d'apporter leur pierre érythréenne, à la suite de « Hannah Arendt, Zbigniew Brzezinki, Carl Friedrich et Raymond Aron» (p.1) et d'une quinzaine d'autres, aux débats à propos du totalitarisme et de l'État totalitaire. Ils font appel, en outre, à Ian Kershaw qui dans, Hitler. Essai sur le charisme en politique, dépasse «la dichotomie classique de 
l'interprétation du totalitarisme entre personnalisme et structuralisme ». Or, dans la préface à l'édition française de ce livre, on lit : «En fait [le concept de totalitarisme] ne dit quasiment rien sur les origines et se borne, pour l'essentiel, à décrire des techniques et des instruments de domination. Finalement, son plus grand mérite est plutôt de mettre en évidence la singularité des systèmes que de souligner leurs similitudes ${ }^{15}$. C'est précisément la question - traitée en seulement 41 pages dans la première partie - des origines de la «dérive » dans laquelle Isayyas entraîne le pays, que les auteurs tentent d'identifier dans deux chapitres: "L'accumulation primitive du pouvoir (1961-1991)» puis dans «La consolidation du pouvoir 1991-2001». Il ressort de ces courts chapitres que la dérive dictatoriale vient de plus loin qu'on l'écrit habituellement. Elle est antérieure à la guerre éthio-érythréenne de 1998-2000, aux lendemains de l'indépendance (1991-1993) et aux premiers soulèvements contre l'annexion à l'Éthiopie (1962).

5 À juste titre, F. Gouéry et J.-B. Jeangène Vilmer insistent sur l'irrésistible ascension d'Isayyas qui, à force de ruse et de cynisme, élimina ses concurrents indépendantistes par la violence. La guérilla est rarement une école de démocratie non violente: l'Érythrée indépendante a accouché dans la douleur. Toutefois, faute de temps, ils reprennent des analyses hâtives démenties par des ouvrages historiques qui relativisent certains passages de leurs deux premiers chapitres. La description de la British Military Administration comme un « âge d'or » est contestée par les historiens ${ }^{16}$. En effet, après avoir songé à y installer des juifs fuyant l'Europe, les Britanniques, après bien des hésitations, remirent l'Érythrée à l'ONU. La coupure, entre musulmans, favorables à l'indépendance (ou au rattachement au Soudan), et chrétiens, partisans de l'union avec l'Éthiopie, était beaucoup moins nette. Ainsi, la Ligue musulmane de l'Ouest opta-t-elle pour la fédération en 1952, tandis que des protestants et des catholiques, soutenus par la communauté italienne et les métis, militaient pour l'indépendance. Les chrétiens de l'Église täwahedo (monophysites et non pas orthodoxes), soutenus par l'Église éthiopienne, rejoignirent le parti unioniste. Les représentants de Haylä Sellasé à Asmära ont vidé de leur substance les institutions de la fédération mais ils n'ont pu agir qu'en jouant des rivalités féroces entre politiciens érythréens ${ }^{17}$. Il est vrai que les Italiens tentèrent de se poser en défenseur de l'islam, mais, sans le large concours de tous les Érythréens (et de Libyens) comme ascari, mais aussi comme dockers, cantonniers, chauffeurs... ils n'auraient jamais conquis l'Éthiopie ${ }^{18}$. Des Érythréens avaient déjà choisi les Italiens à Adwa ${ }^{19}$ et, entre 1936 et 1941, ils entrèrent dans les bandes qui traquaient les résistants, parmi lesquels des Érythréens. Quelques-uns d'entre eux avaient occupé des postes importants en Éthiopie tels Abba Jérôme, qui fut plus que l'informateur de Leiris, Loränso Taezaz, ministre des Affaires étrangères en 1936, et le général Aman Mikaél Andom qui succéda au negus en 1974. Le conflit éthio-érythréen eut (et a ?) une dimension de guerre civile érythréenne tant les liens, et les divisions, entre Éthiopiens et Érythréens, plongent dans l'histoire et la culture et se manifestent par des échanges économiques et des mariages ${ }^{20}$.

6 La deuxième partie, «La dérive », n'appelle guère de réserves et confirme largement les analyses de Gaim. Comme l'écrit Kershaw, cité plus haut, elle « décri[t] des techniques et des instruments de domination». Les auteurs relatent minutieusement l'enfermement (scellement) du pays puis la destruction par atomisation des citoyens en vue de former un « homme nouveau » : nous sommes dans une perspective orwellienne. Par rapport à ce qu'avance Kershaw, ils ne se contentent pas « de mettre en évidence la singularité des systèmes [plutôt] que de souligner leurs similitudes». Dans leurs 
chapitres, ils font la liste des particularités et des similitudes : ainsi, à l'instar de Hitler $^{21}$, Isayyas préfère-t-il l'oral pour faire connaître ses décisions ${ }^{22}$. Pour l'endoctrinement et la mobilisation de la population, Isayyas est le meilleur élève de la Corée du Nord qui bénéficia de la faveur de Mängestu, le dictateur éthiopien. Plus que d'autres tyrans, à l'exception peut-être des Kim ou d'Obiang, il assujettit l'État et l'économie à une étroite coterie dont les membres sont promus et démis selon son bon vouloir : un peu comme en Éthiopie où le negus avait le pouvoir de nommer-démettre (shum sher). Mais, celui qui était chassé de la cour éthiopienne ne disparaissait pas sans laisser de traces comme, en Érythrée, les journalistes et les membres du G 11 ou du G 15 ! Autres ressemblances avec l'URSS de Staline : les chantiers à la «Potemkine » et l'armée purgée, laissée à l'abandon surtout depuis la défaite de 2000. Une garde " prétorienne », bien payée et bien équipée de Tegréens éthiopiens, issus du Démhet ${ }^{23}$, a la charge de protéger le président. Quoique Tegréens en rupture avec l'Éthiopie, ils sont d'abord perçus comme des Éthiopiens, de plus bénéficiant d'avantages matériels. Cette situation paradoxale rappelle la position de la Garde lettone dans les premières années de la Révolution bolchévique. Il y a certes un parti unique, le Front pour la démocratie et la justice (FDJ), copié-collé du FPLE, mais il doit composer avec une division générationnelle entre les tägadelti (anciens combattants) qui, forts de l'expérience du terrain de la guérilla, prennent en charge et surveillent les "héritiers", la nouvelle génération qui n'a pas connu la guerre.

L'analyse de la lutte des générations et de son articulation avec la domestication de la diaspora qui grossit à mesure que le pays se vide de ses jeunes diplômés est sans doute l'apport le plus neuf du livre de F. Gouéry et J.-B. Jeangène Vilmer. Quand on lit ces pages qui retracent l'embrigadement des lycéens dans des camps militaires établis dans les zones les plus reculées du pays, on comprend l'inanité de la distinction qu'on se plait à faire, en Europe, entre «bons » réfugiés politiques et «mauvais » migrants économiques. Depuis que les enseignants et les étudiants de l'université ont demandé des réformes et l'application de la constitution, Isayyas l'a fermée et remplacée par un service militaire obligatoire pour les deux sexes et de durée indéfinie. En fait de diplômes, ils sont " prêtés » aux administrations et aux entreprises où ils reçoivent des salaires de misère qui leur ôtent tout espoir de fonder une famille. Comme l'état d'urgence n'a jamais été levé depuis 1991, toute protestation est passible de peines de prison arbitraires sous la charge de trahison. Et on devient facilement "traître", notamment quand on appartient à une famille éthio-érythréenne ou qui n'a pas milité dans le «bon » front ou dont l'un des membres a quitté le pays. Les différentes polices et garde-frontières tirent sur les fugitifs et extorquent des amendes à leurs parents auxquels, s'il a réussi, on demandera des comptes si le réfugié « dénigre » le régime et ne coopère pas. La diaspora, surveillée par les ambassades, est tenue de verser, tous les ans, $2 \%$ de ses revenus et, au bout de quelques années, pourvu qu'ils soient dociles, les émigrés peuvent, moyennant finance, récupérer un passeport érythréen, acheter un terrain, faire construire et investir au pays. Un racket d'État a été organisé par des officiels qui ont conclu des « marchés » avec les passeurs pour taxer, sous la menace, les familles des migrants des camps du Sinaï et de Libye. Mais, avec plus de 2000 départs par mois, tout contrôle est désormais impossible et l'Érythrée, déjà en faillite économique, se vide de son sang jeune. Tant qu'Isayyas vivra, le régime peut-il s'effondrer? On peut en douter tant les liens sociaux sont brisés par la misère et la répression et tant l'opposition est divisée et instrumentalisée par le pouvoir. Le président est seul : il s'est brouillé avec tous ses voisins auxquels il a fait la guerre et il a 
perdu ses appuis libyens, arabes, iraniens et somaliens. Toutefois, l'UE, l'Égypte et l'Arabie saoudite craignent que l'implosion de l'Érythrée ne produise, sur la rive africaine de la mer Rouge, une conflagration qui s'ajouterait à la menace yéménite. L'Érythrée a proposé que les Européens financent des camps de rétention afin qu'elle garde les migrants sur son sol. Il s'agissait de transposer l'accord Kadhafi-Berlusconi par lequel la Libye était payée pour empêcher les migrants de venir en Europe. Ces négociations ont, heureusement, tourné court car, sûrement, les crédits de l'UE seraient allés, comme les royalties versées par le compagnies minières, dans les poches du président et de son entourage.

8 Ces deux livres se complètent: au premier les images, et au second l'essai de science politique. Toutefois, je maintiens mes réserves à propos d'analyses un peu trop rapides comme dans le chapitre: "La potentielle fracture religieuse ». En fait, l'islam et le christianisme, tant monophysite que protestant ou catholique, sont officiellement reconnus, mais les pentecôtistes, par ailleurs en plein essor comme en Éthiopie, sont pourchassés, comme les ONG, en tant qu'« agents des Américains ». La vraie fracture se situe entre tolérés et interdits, mais tous sont sous la menace. On peut reprendre la question que Raphaël Roigt posait dans son mémoire ${ }^{24}:$ "L'Érythrée, naissance d'une nation, faillite d'un État? » mais en la retournant : « la faillite de l'État compromet-elle la croissance et l'affirmation de la nation érythréenne? » Au moins, en Corée du Nord, on se succède de Kim en Kim, mais en Érythrée qui après Isayyas? Ceux qui ont posé la question croupissent en prison. En terminant la recension de ces livres qui, en dépit de passages parfois faibles, explorent un territoire trop peu connu, je tenterais un rapprochement entre l'Érythrée et l'Algérie. Ces peuples ont, en effet, connu plus qu'une colonisation, une annexion, et ne sont devenus indépendants qu'après un long et sanglant conflit qui a pris des aspects de guerre civile. En outre, des exilés érythréens (pas tous musulmans), des membres du FLN algérien et Abd el-Krim s'étaient rencontrés au Caire. Le Front de libération d'Érythrée (FLE), qui lança la guerre en 1960 et fut défait au cours des années 1970 dans les maquis et dans les camps par le FPLE d'Isayyas, était organisé suivant le modèle du FLN. Ainsi, plus de cinquante ans après l'indépendance de l'Algérie, l'armée s'appuie-t-elle sur les anciens combattants (moudjahidin) pour se maintenir au pouvoir comme Isayyas se maintient en Érythrée grâce aux tägadelti.

9 Les deux livres de F. Gouéry et J.-B. Jeangène Vilmer complètent et renouvellent l'ouvrage fondamental de Gaim Kibreab dont ils sont une très bonne introduction et pour moi, ce n'est pas un mince compliment.

\section{NOTES}

14. C. KUTSCHERA, Érythrée-Eritrea, Paris, Édifra, 1994.

15. I. KeRSHAW, Hitler. Essai sur le charisme en politique, Paris, Folio Histoire, 2013, pp. 4, 12. 
16. G. K. N. TREVASKIS, Eritrea. A Colony in Transition: 1941-52, London, O.U.P., 1960 ; T. NEGASH, Eritrea and Ethiopia: The Federal Experience, Uppsala, Nordiska AfrikainstitutetNew Brunswick, Transaction, 1997.

17. T. MEDHANIE, Eritrea. Dynamics of a National Question, Amsterdam, B.R. Grüner, 1986 ; T. NEGASH (ibid.).

18. I. TADDIA, L'Eritrea-colonia, 1890-1852. Paesaggi, strutture, uomini del colonialismo, Milan, F. Angeli, 1986. T. NEGASH, Italian Colonialism in Eritrea: 1882-1941: Policies, Praxis and Impact, Stockholm, Almqvist och Wiksell, 1987.

19. C. LuCARELl, La huitième vibration, Paris, Métaillé, 2010. A. GASCON \& A. TurCo, « Carlo Lucarelli, La huitième vibration », POUNT, 6, 2012, pp. 187-199.

20. H. ERLICH, Ras Alula and the Scramble for Africa. A Political Biography: Ethiopia and Eritrea 1875-1897, Lawrenceville-Asmara, The Red Sea Press, 1996 ; K. TRONVoll \& T. NEGASH, Brothers at War: Making Sense of the Eritrean-Ethiopian War, Oxford, J. Currey, 2000 ; B. TAFLA, «Eritrea in Retrospect. An Excerpt from the Memoirs of Fitawrari Mika'el Hasama Rakka », Africa (Roma), LX (1), mars 2005, pp. 1-64.

21. I. KERSHAW (2013), op. cit., p. 232.

22. G. KIBREAB, Eritrea. A Dream Deferred, Woodbridge, J. Currey-Nordiska Afrikainstitutet (« Eastern African Series »), 2009.

23. Démokrasyawi menqesqas hezbi Tegray: Mouvement populaire et démocratique du Tegray.

24. R. RoIGT, Travaux et documents sur l'Éthiopie et la corne de l'Afrique, CFEE, CEMAF, Paris 1,3 , avril 2009. 